\title{
Incidence and outcome of cardiopulmonary resuscitation in patients with shunted single ventricle: Advantage of right ventricle to pulmonary artery shunt
}

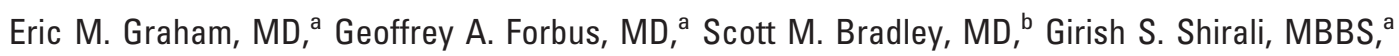

and Andrew M. Atz, MD, ${ }^{a}$ Charleston, SC

$\mathrm{A}$

ppropriate balance among systemic, pulmonary, and coronary blood flow is critical to survival after neonatal palliation in patients with single ventricle physiology. These patients are at risk for circulatory collapse necessitating the use of cardiopulmonary resuscitation (CPR). ${ }^{1}$ Mortality rates are high when CPR is needed. ${ }^{2}$ Although the cause of the mortality has remained elusive, autopsy studies suggest impairment of coronary blood flow as a leading cause. ${ }^{3}$ Greater circulatory stability has been speculated with the right ventricle to pulmonary artery shunt (RV-PA) in comparison with a modified Blalock-Taussig shunt (BTS). ${ }^{4}$ A purported advantage of the RV-PA is elimination of diastolic runoff, with possible improved coronary perfusion. The differences between these 2 surgical approaches on the incidence and outcome of cardiac arrest in patients requiring CPR were investigated.

\section{Clinical Summary}

Between January 2000 and March 2005, 112 patients with single ventricle physiology underwent palliation with a BTS $(n=75)$ or an RV-PA $(n=37)$. Given the prevalence of preexisting coronary anomalies, patients with pulmonary atresia with intact ventricular septum were excluded. The Norwood procedure was the initial palliative surgery in 45 patients $(60 \%)$ with a BTS and all 37 patients $(100 \%)$ with an RV-PA. Patients underwent surgery concurrently; however, the majority of patients requiring a Norwood procedure after June 2002 received a RV-PA. Patient courses were reviewed and data were compared for all in-hospital cardiac arrests requiring CPR with chest compressions from the time of initial palliation until second-stage palliation. Consistent with the recommended guidelines for conducting research on in-hospital resuscitation, cardiac arrest was defined as chest compressions or the absence of a palpable spontaneous pulse that was not resolved with only airway intervention. ${ }^{5}$ Patients in whom extracorporeal membrane oxygenation (ECMO) was prophylactically used to prevent

From the Divisions of Pediatric Cardiology ${ }^{\mathrm{a}}$ and Cardiothoracic Surgery, ${ }^{\mathrm{b}}$ Medical University of South Carolina, Charleston, SC.

Received for publication Nov 18, 2005; accepted for publication Dec 22, 2005.

Address for reprints: Andrew M. Atz, MD, Medical University of South Carolina, 165 Ashley Avenue, PO Box 250915, Charleston, SC 29425 (E-mail: atzam@musc.edu).

J Thorac Cardiovasc Surg 2006;131:e7-8

$0022-5223 / \$ 32.00$

Copyright $\odot 2006$ by The American Association for Thoracic Surgery

doi:10.1016/j.jtcvs.2005.12.028 cardiac arrest were not included. Patients were considered successfully resuscitated if they survived at least 24 hours after cardiac arrest. $^{5}$

Comparison between groups was performed with Fischer exact test, chi-square test, or Wilcoxon rank-sum test as appropriate.

There were 22 of 112 patients $(20 \%)$ who had any $(\geq 1)$ in-hospital cardiac arrest requiring CPR. The incidence was not different between groups: BTS $n=15 / 75$ (20\%), RV-PA $n=7 / 37$ $(19 \%), P=.91$. There were 27 separate CPR events among these 22 patients (Figure 1). Twelve CPR events occurred in the RV-PA group: 1 patient with 2 events and 2 patients with 3 events between 16 hours and 173 days from initial arrest. Of these, 2 ultimately died. There were no repeat events in the BTS group. Patients with an RV-PA had improved survival after CPR compared with those with a BTS (Figure 2). When we compared only patients who underwent the Norwood procedure, CPR was successful in $25 \%$ of patients (2/8) with a BTS and $67 \%$ of patients $(8 / 12)$ with an RV-PA $(P=.17)$. Among patients demonstrating cardiac arrest, survival to hospital discharge for both groups was $33 \%$ with a trend toward improved hospital survival in the RV-PA group (56\%) compared with the BTS group $(20 \%)(P=.1)$.

Our institution does not use a rapid-resuscitation ECMO circuit as a component of routine resuscitation management. ECMO was used in 2 patients with a BTS who remained in critical condition immediately after a CPR event. Both patients died once ECMO support was withdrawn, and both deaths were considered as "CPR deaths."

\section{Discussion}

It has been reported that $6 \%$ of infants who underwent congenital heart surgery of all types sustained a documented cardiac arrest with a $41 \%$ survival to hospital discharge. ${ }^{2}$ The current study shows the incidence of CPR in a select group of patients with a shunted single ventricle to be $20 \%$ after initial palliation with a $33 \%$ survival to discharge. Patients with an RV-PA had improved survival after CPR events compared with those with a BTS. The RV-PA shunt origin from the single ventricle may allow for comparatively more effective pulmonary blood flow during chest compressions.

The limitations of this study are related primarily to the inherent problems of a cohort study design. The comparison groups, although more homogenous than any series previously reported, ${ }^{2}$ were not equally balanced in terms of underlying anatomic diagnosis. In addition, the cause of cardiac arrest in these patients and whether the cause of and, thus response to, CPR may differ early versus later after initial palliation remain unclear. Nevertheless, despite a larger proportion of patients receiving a Norwood palli- 


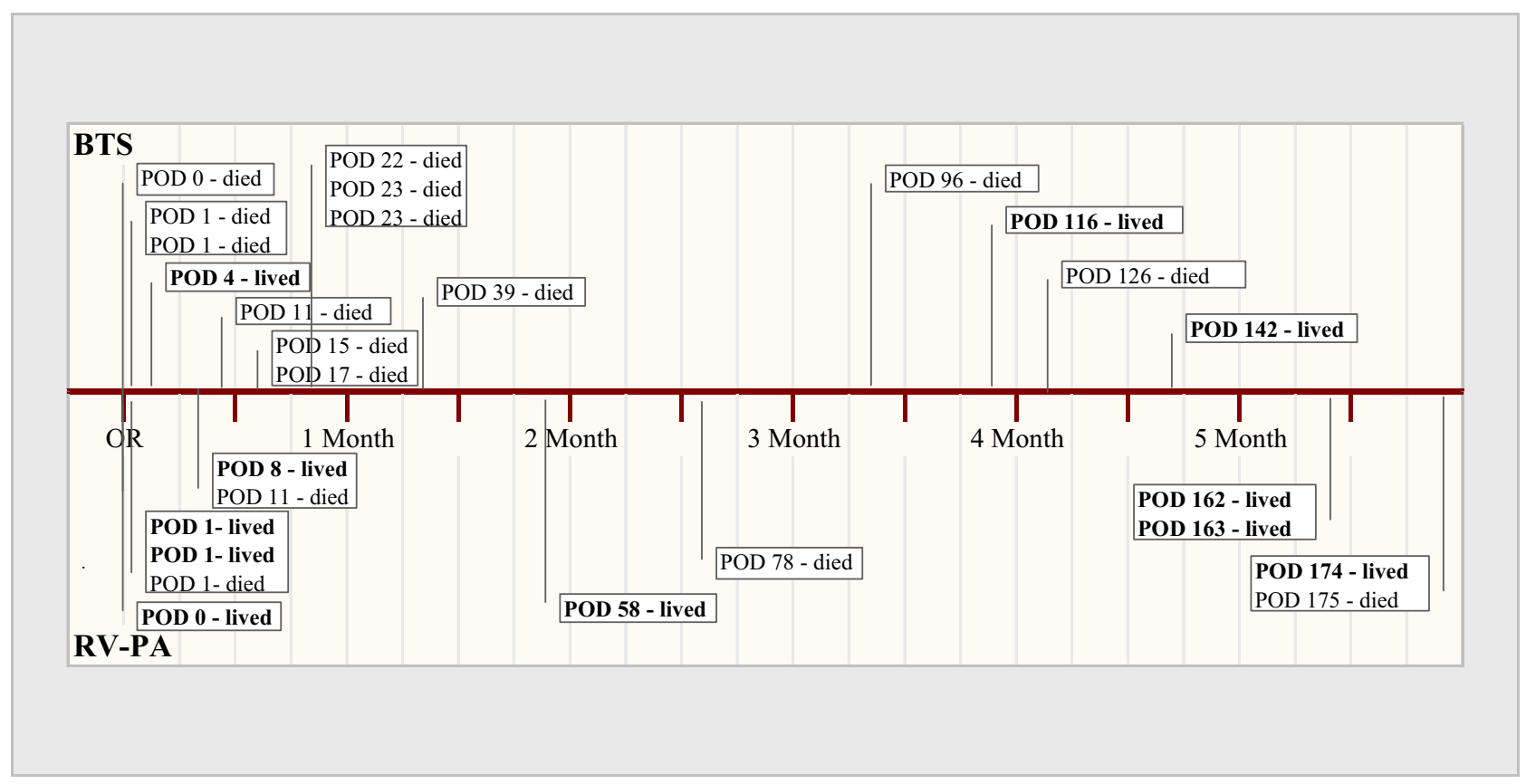

Figure 1. Episodes of cardiac arrest requiring CPR in patients with a BTS (top) and an RV-PA (bottom). Time of arrest was not different between groups; median POD 22 for BTS and 35 for RV-PA (P=.75). BTS, Blalock-Taussig shunt; $R V-P A$, right ventricle to pulmonary artery shunt; $P O D$, postoperative day.

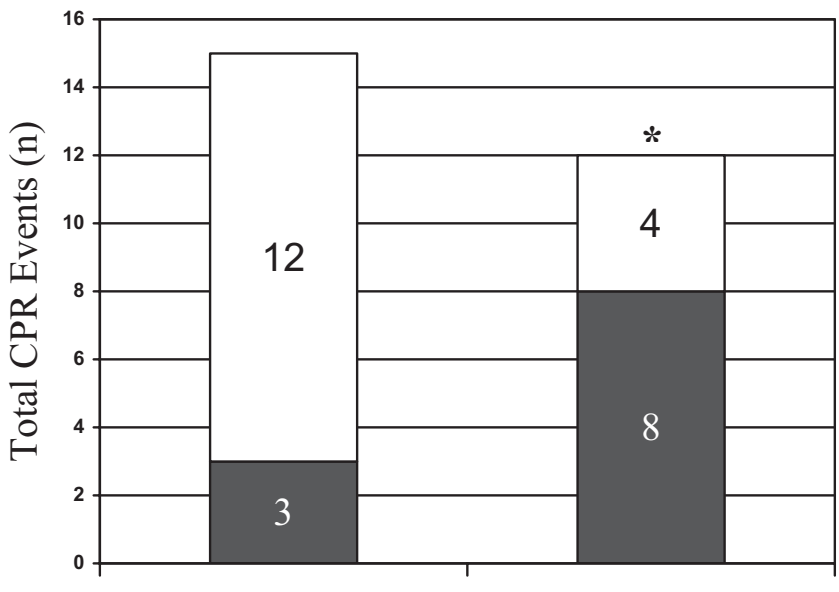

BTS

Type of Shunt

Figure 2. Successful resuscitation after cardiac arrest was greater after palliation with an RV-PA (8/12) than a BTS (3/15); ${ }^{*} P=.02$. Survived (filled squares); died (empty squares). CPR, Cardiopulmonary resuscitation; BTS, Blalock-Taussig shunt; $R V$ $P A$, right ventricle to pulmonary artery shunt.

ation in the RV-PA group, the frequency of CPR events was not different between shunt types. A true comparison between groups will require a prospective comparison of shunt types in a uniform population. Enrollment has commenced in a multicenter trial from the Pediatric Heart Network, which will greatly contribute to answering outstanding questions.

\section{References}

1. Fenton KN, Siewers RD, Rebovich B, Pigula FA. Interim mortality in infants with systemic-to-pulmonary artery shunts. Ann Thorac Surg. 2003;76:152-7.

2. Rhodes JF, Blaufox AD, Seiden HS, Asnes JD, Gross RP, Rhodes JP, et al. Cardiac arrest in infants after congenital heart surgery. Circulation. 1999;100(Suppl II):II194-9.

3. Bartram U, Grunenfelder J, Van Praagh R. Causes of death after the modified Norwood procedure: a study of 122 postmortem cases. Ann Thorac Surg. 1997;64:1795-802.

4. Pizarro C, Mroczek T, Malec E, Norwood WI. Right ventricle to pulmonary artery conduit reduces interim mortality after stage 1 Norwood for hypoplastic left heart syndrome. Ann Thorac Surg. 2004;78: 1959-64.

5. Cummins RO, Chamberlain D, Hazinski MF, Nadkarni V, Kloeck W, Kramer E, et al. Recommended guidelines for reviewing, reporting, and conducting research on in-hospital resuscitation: the in-hospital 'Utstein style'. Circulation. 1997;95:2213-39. 